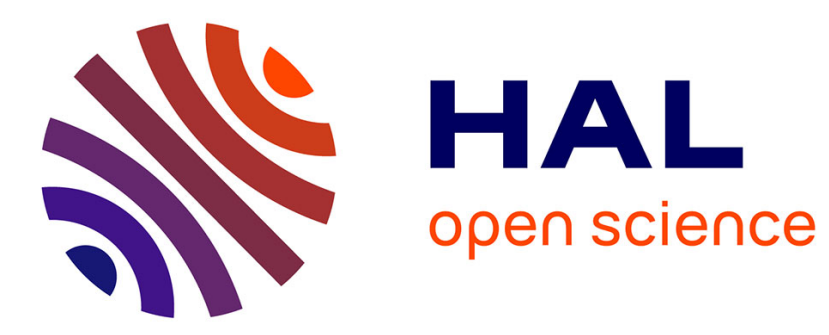

\title{
Spectrophotomètre double faisceau à balayage d'image pour ultracentrifugeuse analytique
}

\author{
J. Neimark, J. Pouyet
}

\section{To cite this version:}

J. Neimark, J. Pouyet. Spectrophotomètre double faisceau à balayage d'image pour ultracentrifugeuse analytique. Revue de Physique Appliquée, 1970, 5 (6), pp.877-884. 10.1051/rphysap:0197000506087700 . jpa-00243468

\section{HAL Id: jpa-00243468 https://hal.science/jpa-00243468}

Submitted on 1 Jan 1970

HAL is a multi-disciplinary open access archive for the deposit and dissemination of scientific research documents, whether they are published or not. The documents may come from teaching and research institutions in France or abroad, or from public or private research centers.
L'archive ouverte pluridisciplinaire HAL, est destinée au dépôt et à la diffusion de documents scientifiques de niveau recherche, publiés ou non, émanant des établissements d'enseignement et de recherche français ou étrangers, des laboratoires publics ou privés. 


\title{
SPECTROPHOTOMÈTRE DOUBLE FAISCEAU A BALAYAGE D'IMAGE POUR ULTRACENTRIFUGEUSE ANALYTIQUE
}

\author{
J. NEIMARK et J. POUYET \\ Centre de Recherches sur les Macromolécules, Strasbourg
}

(Reçu le 3 août 1970, révisé le 29 septembre 1970)

\begin{abstract}
Résumé. - Description d'un spectrophotomètre double faisceau, à balayage d'image, adapté à une ultracentrifugeuse analytique. Des voltmètres de crête à recyclage synchrone confèrent à ce spectrophotomètre une réponse en Densité Optique indépendante de la vitesse de rotation entre 1500 et 67000 tours par minute. La lecture sur l'enregistreur est linéaire en Densité Optique. La précision de lecture est de $\pm 0,002$.

Le domaine spectral utile s'étend de 700 à $230 \mathrm{~nm}$.
\end{abstract}

Abstract. - A description is given of a double beam spectrophotometer for the studies with an analytical ultracentrifuge. Peak voltmeters with synchronous recycling give an output, linear in Optical Density, independant of the speed of rotation between 1500 and $67000 \mathrm{R}$. P. M. The accuracy is \pm 0.002 unit $\mathrm{O}$. $\mathrm{D}$.

The usable range of wavelength is between 700 and $230 \mathrm{~nm}$.

La grande sensibilité de la mesure des concentrations par absorption de la lumière a suscité un développement considérable de cette technique dans le domaine de l'ultracentrifugation, et plus spécialement dans l'étude des polymères d'origine biologique, tels les acides nucléiques et les protéines.

A l'heure actuelle, les ultracentrifugeuses analytiques sont en majorité équipées d'un système optique pour la mesure de l'absorption de la lumière par la méthode photographique : la plaque photographique constitue en effet un convertisseur logarithmique simple, mais dont l'utilisation impose des servitudes très lourdes pour un résultat généralement peu satisfaisant, surtout dans le cas des équilibres de sédimentation.
H. Schachman, dans le but d'apporter à la technique de l'ultracentrifugation les avantages incontestables des mesures précises d'absorption de la lumière (grande sensibilité et pouvoir d'analyse élevé dans le cas de systèmes à multiples composants), a étudié et développé un spectrophotomètre double faisceau adapté à l'ultracentrifugeuse analytique $[1,2]$.

Nous présentons ici la réalisation originale que nous avons mené à bien sur une centrifugeuse du C. R. M.

Description du spectrophotomètre. - Le principe est, en tous points, identique à celui adopté par H. Schachman mais la réalisation a été conduite de façon différente. Le diagramme général (Fig. 1) montre

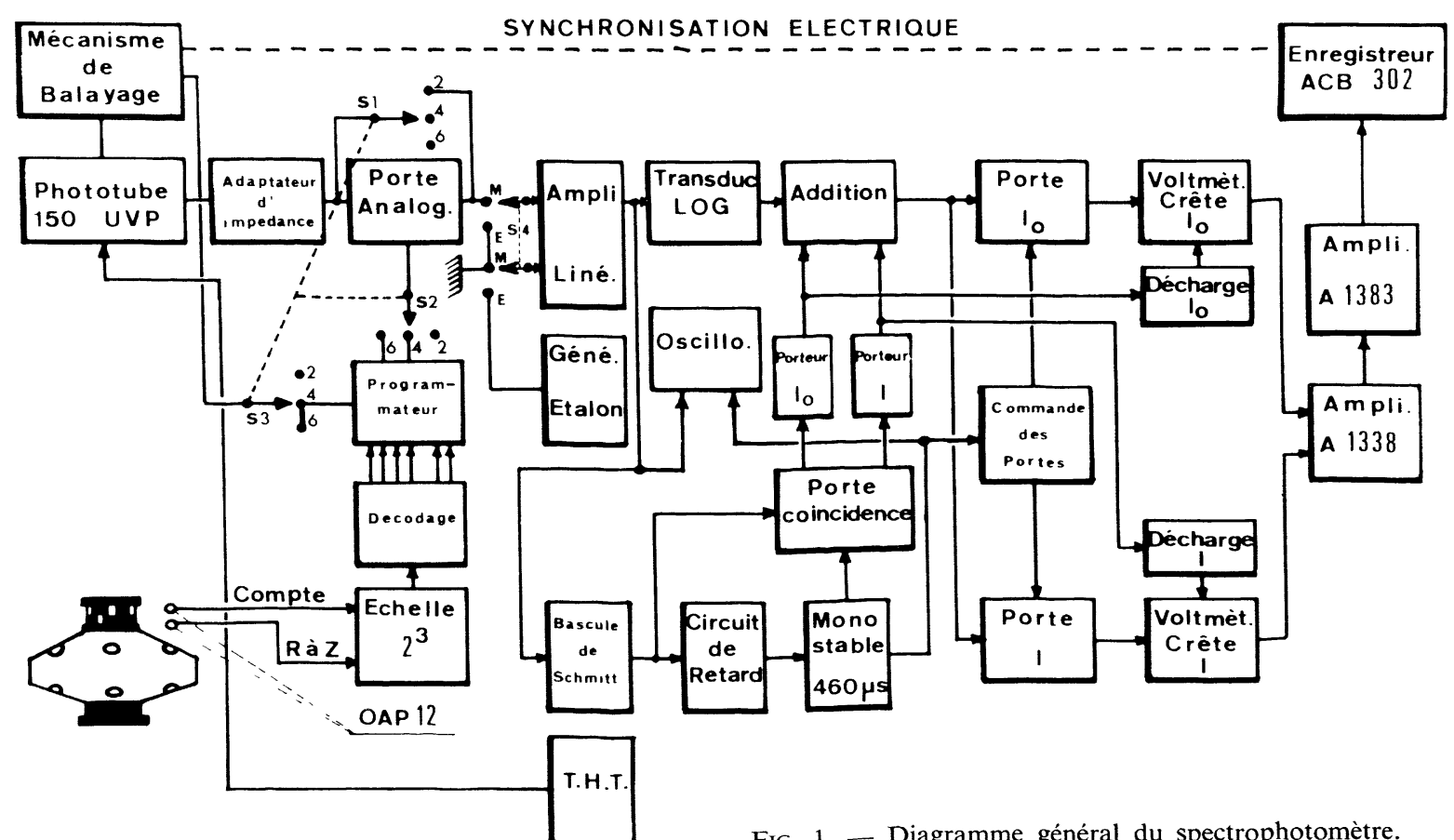

FIG. 1. - Diagramme général du spectrophotomètre. 
l'ensemble des circuits électroniques et leurs interconnections.

Nous décomposerons la description de ce montage en quatre parties :

A. - Ensemble des circuits de mesure ;

B. - Ensemble des circuits de synchronisation;

C. - Montage optique ;

D. - Circuit multiplex ;

puis nous présenterons brièvement les résultats expérimentaux obtenus avec ce spectrophotomètre.

A. Ensemble des circuits de mesure. - Le tracé de la courbe de distribution de la concentration à l'intérieur d'une cellule de centrifugation impose de mesurer en chaque point d'abscisse $R, R$ désignant la distance d'un point de la cellule à l'axe de rotation du rotor, l'intensité de la lumière incidente $I_{0}$ et l'intensité de la lumière transmise $I$. L'utilisation d'une cellule double secteur dont l'un contient le solvant et l'autre la solution permet de déterminer les deux intensités $I_{0}$ et $I$.

La valeur de la densité optique en fonction de la distance à l'axe de rotation est donnée par la relation :

et :

$$
\mathrm{DO}(R)=\log I_{0}(R)-\log I(R)
$$

$$
C(R)=\frac{\mathrm{DO}(R)}{K_{\lambda} d}
$$

$K_{\lambda}$ et $d$ sont les coefficients habituels de la loi de BeerLambert.

1. Amplificateur linéAire. - Cet amplificateur, différentiel, reçoit sur une de ses entrées (position Mesure) les deux impulsions $I_{0}$ et $I$ recueillies aux bornes de la résistance de charge d'un photomultiplicateur 150 UVP (Radiotechnique). L'autre entrée (position Etalonnage) reçoit les impulsions d'un générateur Etalon en Densité Optique. Le gain peut être réglé entre 30 et 3000 . Le signal de sortie attaque le transducteur logarithmique et la chaîne de synchronisation.

2. Transducteur logarithmique. - De préférence au montage à diode, ce circuit, basé sur la relation entre la tension base-émetteur $V_{\mathrm{EB}}$ et le courant émetteur $I_{\mathrm{E}}$ d'un transistor, a été établi en tenant compte des considérations développées par G. Gianelli et L. Stanchi [3] et J. P. Vautrin [4].

La figure 2 représente la caractéristique dynamique, en impulsions, relevée sur ce circuit et qui fait apparaître une relation logarithmique, entre les tensions d'entrée et de sortie, s'étendant sur 2,5 décades environ.

3. Additionneur. - Ce circuit permet de placer les impulsions du signal, à l'aide de deux impulsions carrées synchrones ( linéaire des caractéristiques des circuits suivants. Le zéro électrique, donc la ligne de base correspondant à une Densité Optique nulle, est obtenu par un réglage fin différentiel de l'amplitude des «Porteurs».

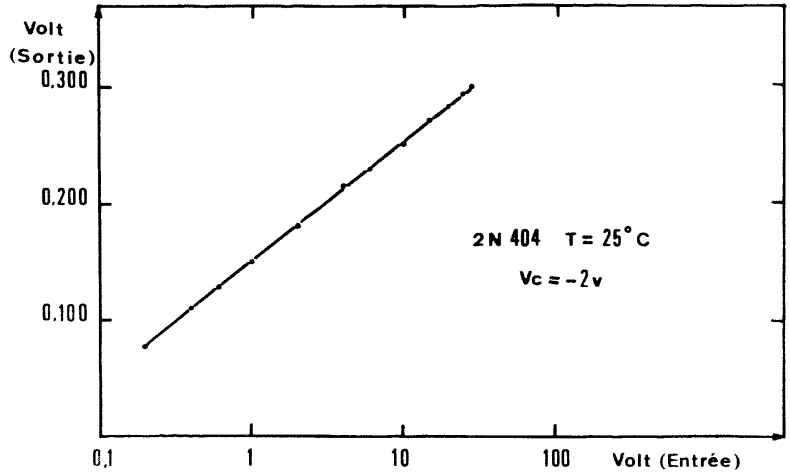

FIG. 2. - Caractéristique dynamique, en impulsion, du transducteur logarithmique. Le temps de montée des impulsions est de 50 ns.

4. Portes Électroniques. - Les impulsions «Référence » $\left(\log I_{0}\right)$ sont séparées des impulsions « Mesure » $(\log I)$ par une double porte à diodes, linéaire, sans seuil. La tension de commande n'apparaît pas dans les signaux de sortie en raison de la configuration symétrique des portes dont le gain, de ce fait, est de 0,5 .

A la sortie des portes les impulsions « Référence » et les impulsions « Mesure » sont appliquées aux voltmètres assurant la mémorisation de la tension de crête de chacune des impulsions du signal.

5. Voltmètres DE CRÊTE. - Les deux secteurs de la cellule de mesure ont une ouverture angulaire de $2^{\circ} 30^{\prime}$. La durée totale d'une impulsion du signal est de $2^{\circ} 30^{\prime} / 360^{\circ}$, soit $7 / 1000$ environ de la durée d'une révolution du rotor et la durée utile du signal est environ égale à la moitié de la durée totale d'une impulsion. Il faut donc mémoriser l'amplitude de crête de chaque impulsion durant la quasi-totalité de la durée de révolution du rotor.

La constante de temps adoptée pour le circuit de mémorisation est de $12 \mathrm{~s}:$ la décroissance de la tension mémorisée n'excède alors pas $2 / 1000$ de la tension de crête pour une durée de $30 \mathrm{~ms}$ correspondant à la vitesse de rotation la plus faible $(2000 \mathrm{t} / \mathrm{mn})$. Mais cette valeur est relativement grande devant la durée d'exploration de l'image de la cellule (15 à $30 \mathrm{~s}$ ). Afin d'obtenir un enregistrement rapide de toutes les variations de l'intensité lumineuse, les condensateurs de «mémorisation » de chacune des voies « Référence » et « Mesure » sont déchargés complètement par une impulsion très brève ( $<100 \mathrm{~ns})$ synchronisée par le début des impulsions correspondantes du signal. Le montage réalisé est un voltmètre à recyclage synchrone [5].

Il est ainsi possible de mettre en évidence une variation de 1 unité de D. O. en $1 \mathrm{~ms}$ à $60000 \mathrm{t} / \mathrm{mn}$ et en $30 \mathrm{~ms}$ à $2000 \mathrm{t} / \mathrm{mn}$.

La fente d'exploration de l'image présente une largeur, ramenée au niveau de la cellule de mesure, de $0,0030 \mathrm{~cm}(30 \mu)$ : le gradient maximal $\mathrm{dDO} / \mathrm{d} R$ susceptible d'être mesuré est de $300 \mathrm{~cm}^{-1}$. Des gradients de cette importance ne se rencontrent jamais, ni 
dans le cas de mesure de vitesse de sédimentation, ni « a fortiori » dans le cas d'équilibre de sédimentation. La définition du tracé de la courbe DO $f(R)$ est donc très élevée même dans le cas de soluté sédimentant très vite et présentant un coefficient de diffusion très faible (virus par exemple).

6. AMPlificATEUR DifFÉRENTIEL. - AMPlifiCATEUR DE PUISSANCE. - L'opération $\left(\log I_{0}-\log I\right)$ est effectuée par un amplificateur différentiel Rochar A 1338. Le gain est ajustable entre 20 et 3000 . La sortie de cet amplificateur attaque un amplificateur de puissance Rochar A 1383 dont le courant de sortie commande l'oscillographe rapide d'un enregistreur ACB 302.

Les courbes $\mathrm{DO} f(R)$ sont obtenues sur papier à noircissement direct. Un marquage de temps

$$
\left(1 \times 10^{-2}, 1 \times 10^{-1} \text { et } 1 \mathrm{~s}\right)
$$

permet de contrôler la vitesse de défilement du papier.

Le bruit de fond résiduel de la chaîne électronique est de $3 \times 10^{-3}$ volt : pour une tension nominale $I_{0}$ de 25 volts, ce bruit est équivalent à une Densité Optique de $4($ Signal/bruit $=80 \mathrm{db})$.

La sortie de l'amplificateur de puissance est munie de filtres passe-bas dont les caractéristiques confèrent à l'enregistreur des constantes de temps égales à $6 \times 10^{-2}$ et $12 \times 10^{-2}$ s utilisées lors des mesures de $S$ et de $16 \times 10^{-2}$ et $25 \times 10^{-2}$ s utilisées lors des mesures d'équilibre de sédimentation.

L'ensemble des circuits "Transducteur logarithmique, Voltmètres de crête et Amplificateurs de sortie » présente des caractéristiques indépendantes de la vitesse de rotation.

7. GÉNÉRATEUR ÉTALON. - Le générateur est conçu pour «mimer» le signal réel correspondant à une vitesse de rotation de $30000 \mathrm{t} / \mathrm{mn}$ : les deux impulsions de $11 \mu$ s sont séparées par un intervalle de temps identique.

Un atténuateur général permet de faire varier simultanément les amplitudes des deux impulsions, leur rapport restant constant.

Un atténuateur logarithmique permet de régler l'amplitude de la seconde impulsion à des valeurs correspondant aux Densités Optiques données dans le tableau suivant :

\begin{tabular}{|c|c|c|c|c|}
\hline Position & $E_{\text {volt. }}$ & $E_{0} / E$ & $\log E_{0} / E$ & D. $\mathrm{O}$. \\
\hline 1 & 1,63104 & 1 & 0 & 0 \\
\hline 2 & 1,29561 & 1,25889 & 0,09999 & 0,1 \\
\hline 3 & 1,02900 & 1,58507 & 0,20003 & 0,2 \\
\hline 4 & 0,81758 & 1,99496 & 0,29994 & 0,3 \\
\hline 5 & 0,64944 & 2,51145 & 0,39992 & 0,4 \\
\hline 6 & 0,40944 & 3,98358 & 0,60027 & 0,6 \\
\hline 7 & 0,25802 & 6,32137 & 0,80008 & 0,8 \\
\hline 8 & 0,16345 & 9,97883 & 0,99908 & 1,0 \\
\hline 9 & 0,12982 & 12,56385 & 1,09900 & 1,1 \\
\hline 10 & 0,08183 & 19,93205 & 1,29955 & 1,3 \\
\hline 11 & 0,05173 & 31,52986 & 1,49872 & 1,5 \\
\hline
\end{tabular}

La figure 3 reproduit une courbe d'échelonnage obtenue sur l'enregistreur à partir des signaux du générateur.

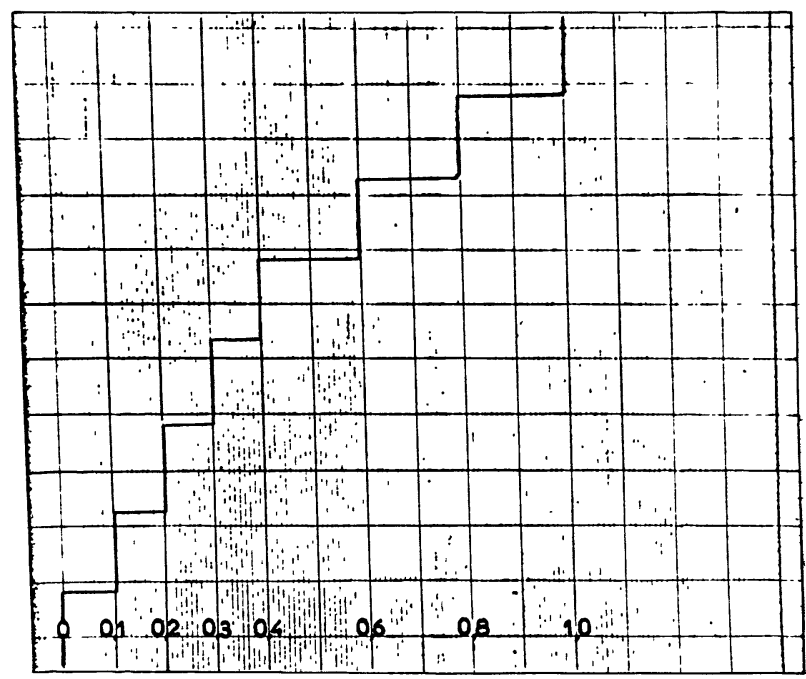

FIG. 3. Reproduction d'un enregistrement d'une courbe d'étalonnage en densité optique. La vitesse de défilement du papier est de $1 \mathrm{~cm} / \mathrm{s}$.

L'atténuateur général nous donne la possibilité d'explorer la caractéristique du convertisseur logarithmique : après avoir affiché une valeur de D. O., 0,3 ou 0,4 par exemple, la déviation du spot reste constante et égale à la valeur choisie $(\Delta \mathrm{D} . \mathrm{O} .<0,005)$ pour une variation de $\mathrm{I}_{0}$ correspondant àune Densité Optique de 1, c'est-à-dire en atténuant les deux impulsions par un facteur 10 .

La stabilité obtenue au niveau du transducteur logarithmique et de voltmètres de crête est suffisamment bonne pour augmenter le gain de l'amplificateur différentiel A 1338 et obtenir la déviation totale de l'enregistreur pour une Densité Optique de 0,1 . La précision obtenue est alors de $0,1 \pm 0,002$.

B. Ensemble des circuits de synchronisation. - Le diagramme 4 montre les relations entre les impulsions du signal et les différentes impulsions mises en jeu pour la séparation des impulsions «Référence» et « Mesure».

Les impulsions « Porteurs » sont obtenues par séparation des deux impulsions de la Bascule de Schmitt.

Les impulsions de décharge des voltmètres de crête sont obtenues par différentiation du front de montée des «Porteurs». L'univibrateur de commande des portes est déclenché à partir du front arrière de la première impulsion de la bascule de Schmitt par l'intermédiaire d'un circuit de retard ajustable entre 1 et $200 \mu s$.

C. Montage optique et mécanisme d'exploration de l'image. - Le monochromateur Bausch et Lomb, à réseau, directement monté sur la barricade de la cen- 


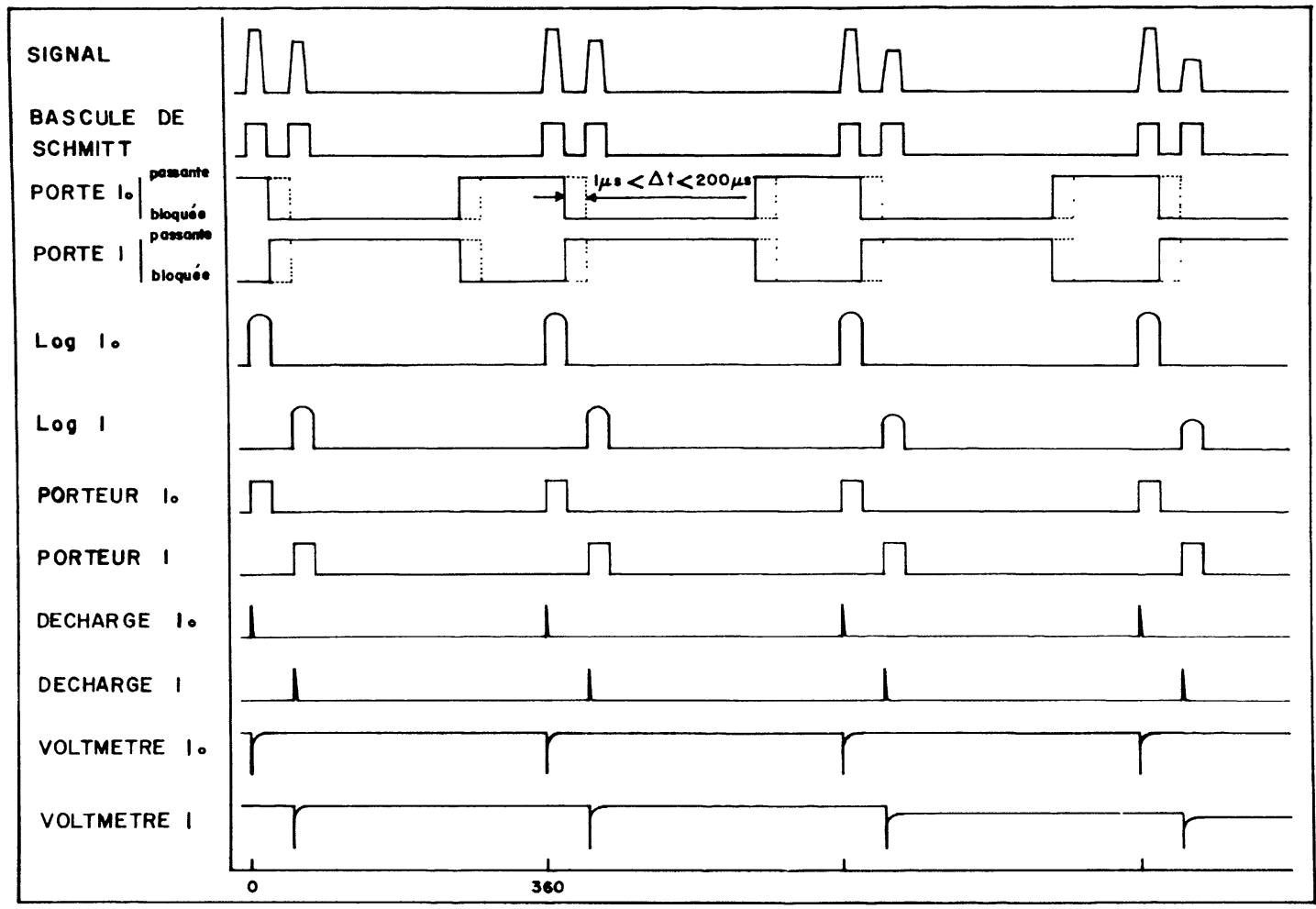

Fig. 4. - Diagramme général des impulsions de synchronisation et de séparation des deux impulsions du signal.

trifugeuse, couvre le domaine des longueurs d'onde UV-Visible (200 à $880 \mathrm{~nm}$ ).

Nous avons vérifié que le rapport lumière utile $I \lambda \mathrm{u}$ sur la lumière parasite $I \mathrm{p}$, compte tenu du rapport de la sensibilité maximale de la photocathode $S$ max à la sensibilité pour la longueur d'onde de la lumière utile $S \lambda \mathrm{u}$, est toujours supérieur à 1000 .

$$
\frac{I \lambda \mathrm{u}}{I \mathrm{p}} \times \frac{S \lambda \mathrm{u}}{S \max }>1000
$$

La lampe est une lampe au Xénon de 450 watts (OSRAM X BO 450).

Le montage optique est schématisé par la figure 5 .

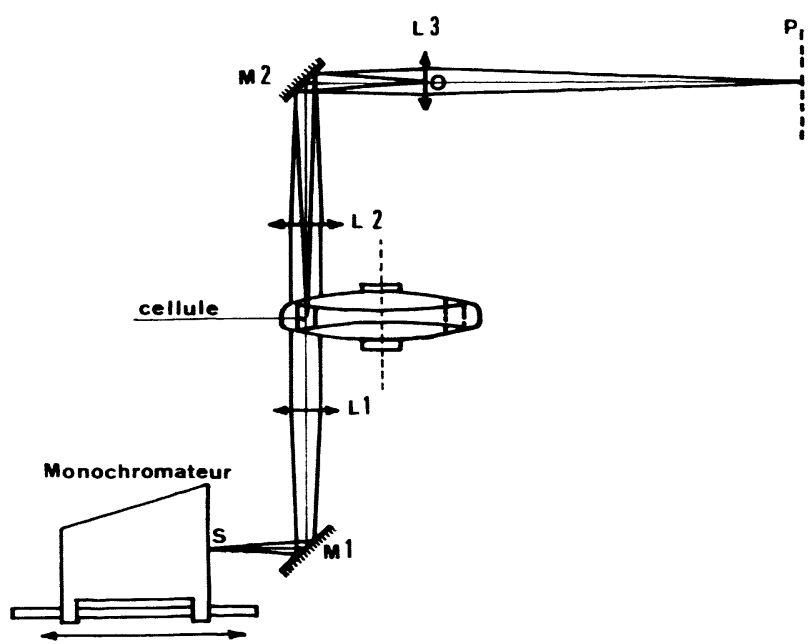

FIG. 5. - Schéma général du système optique.
La lentille $\mathrm{L}_{3}$ forme l'image de la cellule dans le plan $\mathbf{P}$ contenant la fente d'exploration placée devant la photocathode du tube 150 UVP. En fait 3 lentilles, montées sur un manège tournant (Fig. 6), sont utilisées pour couvrir tout le domaine spectral.

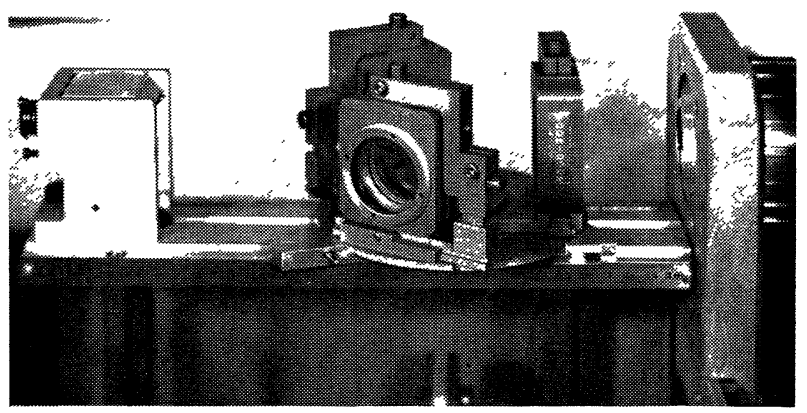

Fig. 6. - Photographie du manège des 3 lentilles $\mathrm{L}_{3}$. A gauche, on voit le miroir $\mathrm{M}_{2}$. A droite, on distingue l'orifice du tubecaméra.

La fente d'exploration de l'image a une hauteur de $3 \mathrm{~m} / \mathrm{m}$ et une largeur de 100 microns. La fente peut être orientée avec précision dans la direction perpendiculaire au champ de gravitation. Le déplacement du chariot portant le phototube est assuré par un moteur synchrone. Un train d'engrenage permet d'obtenir des temps d'exploration de l'image de $5,10,15$ et $30 \mathrm{~s}$.

D. Circuit multiplex. - Le repérage des différentes cellules est assuré par des zones réfléchissantes dessi- 


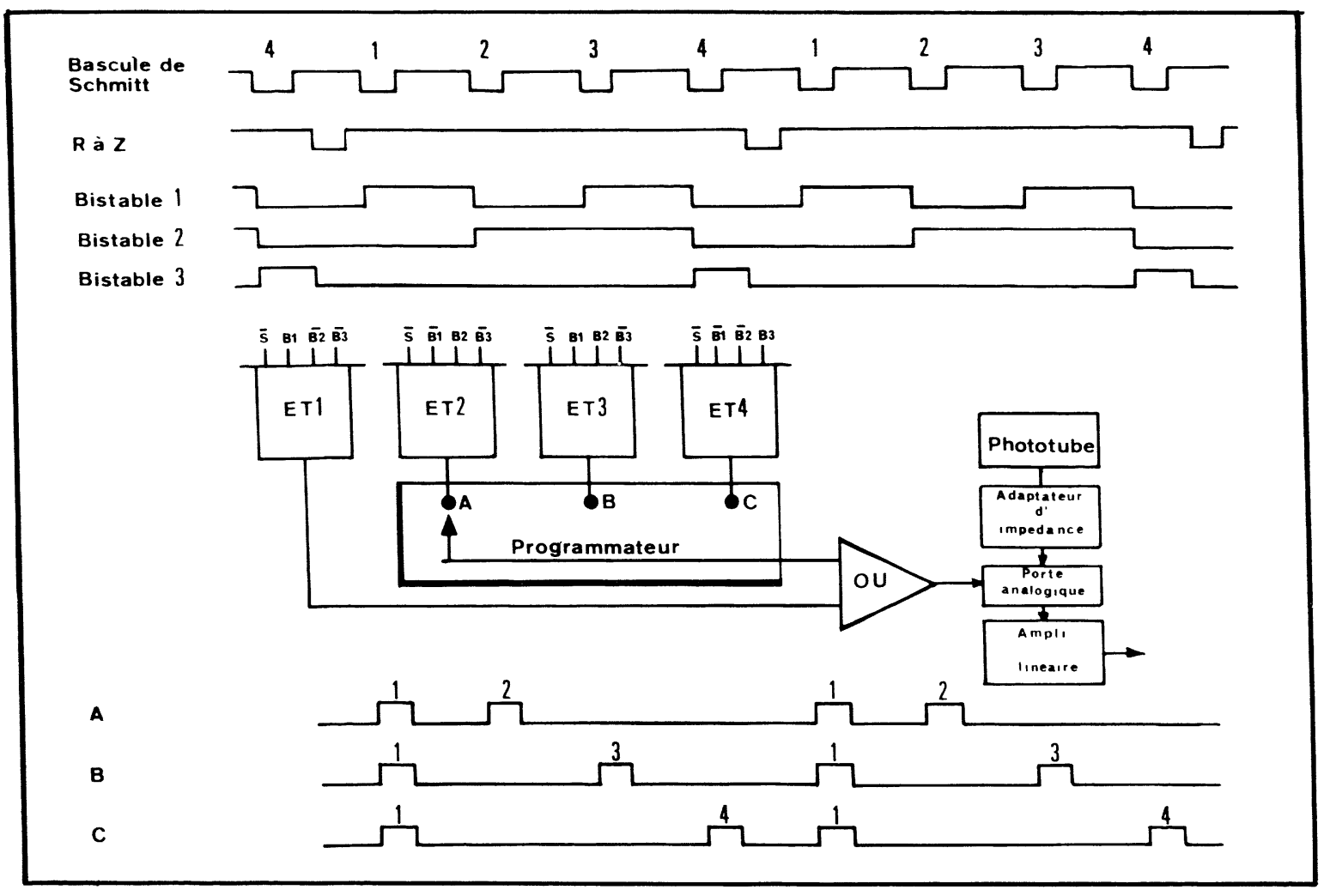

FIG. 7. - Diagramme des impulsions et des circuits logiques du multiplexeur.

nées sur la moitié supérieure de la colerette des rotors et donnant des impulsions lumineuses lues par une photodiode OA P 12. Une bascule de Schmitt synchronisée par le signal de la photodiode délivre des impulsions carrées comptées par une échelle de 8.

A chaque tour du rotor, une zone réfléchissante dessinée sur la moitié inférieure de la colerette remet l'échelle de 8 à 0 .

Le signal de commande de la porte analogique du multiplex est élaboré par l'intersection des niveaux logiques de la bascule de Schmitt et des bistables de l'échelle 8 .

Un programmateur pas à pas, Saelectroswitch, sélectionne successivement chacune des cellules d'un rotor à 4 ou 6 logements (Fig. 7).

E. Résultats expérimentaux. - Le spectrophotomètre que nous venons de décrire est en service sur une ultracentrifugeuse analytique SPINCO E du C. R. M. depuis la fin de l'année 1967. Le circuit multiplexeur a été installé dans le courant de l'année 1968.

L'ensemble des circuits électroniques reste constamment sous tension pour maintenir l'équilibre thermique par rapport à la température ambiante et minimiser ainsi toutes les dérives d'origine thermique.

Un unique contrôle de la linéarité du transducteur logarithmique et du gain de l'amplificateur différentiel A 1338, en début d'expérience, est suffisant pour les mesures de vitesse de sédimentation et les détermina- tions des coefficients de diffusion, mesures dont la durée peut atteindre trois à quatre heures.

Depuis la mise en service, nous n'avons pas changé un seul transistor et la maintenance se réduit à une vérification annuelle des tensions d'alimentation, au réglage de diverses tensions de compensation ainsi qu'au contrôle du gain unité et à la linéarité des deux voltmètres de crête.

Le domaine des longueurs d'onde réellement utilisable s'étend de $600 \mathrm{~nm}$ à $230 \mathrm{~nm}$. Des modifications de l'optique sont actuellement en cours pour étendre les possibilités de mesure jusqu'à $220 \mathrm{~nm}$.

Les résultats expérimentaux obtenus jusqu'à présent couvrent pratiquement tous les domaines accessibles à l'ultracentrifugation :

1. EQUILIBRE EN GRADIENT DE DENSITÉ DE CHLORURE DE CÉSIUM. - La figure 8 montre un enregistrement obtenu suivant cette méthode.

2. ETUde D'UNe PROTÉINe. - Mesure de la constante de sédimentation, du coefficient de diffusion et de la masse moléculaire par équilibre de sédimentation.

Cette protéine est une fraction d'histone spécifique aux érythrocytes de poulet.

Les figures 9,10 et 11 montrent, respectivement, les courbes de distribution intégrale de concentration dans le cas d'une mesure de constante de sédimentation, 4 des enregistrements réalisés au cours d'une mesure du 


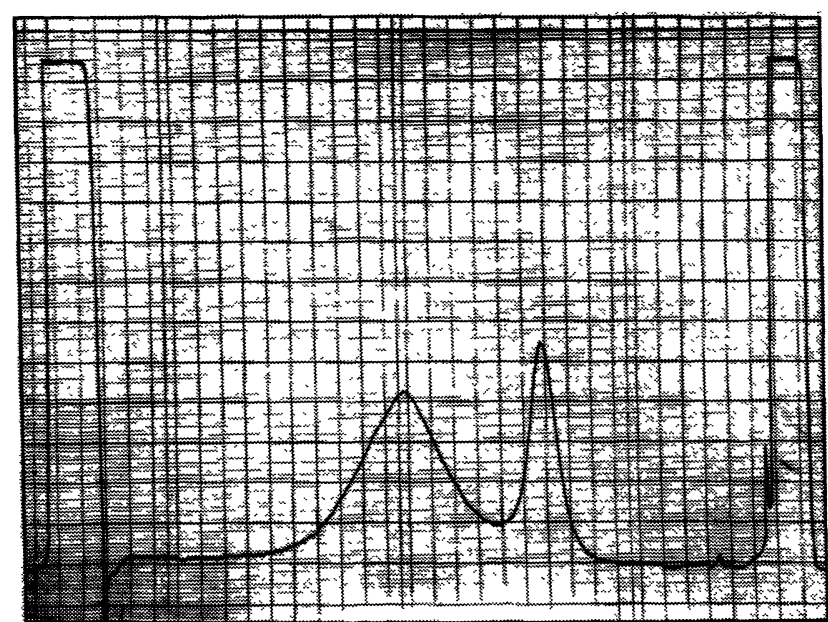

Fig. 8. - Enregistrement d'un équilibre de sédimentation en gradient de densité $\mathrm{CsCl}$.

Substance : ADN

Vitesse : $44770 \mathrm{t} / \mathrm{mn}$.

Température : $20^{\circ} \mathrm{C}$.

Longueur d'onde : $260 \mathrm{~nm}$.

Durée d'exploration de l'image : $32 \mathrm{~s}$.

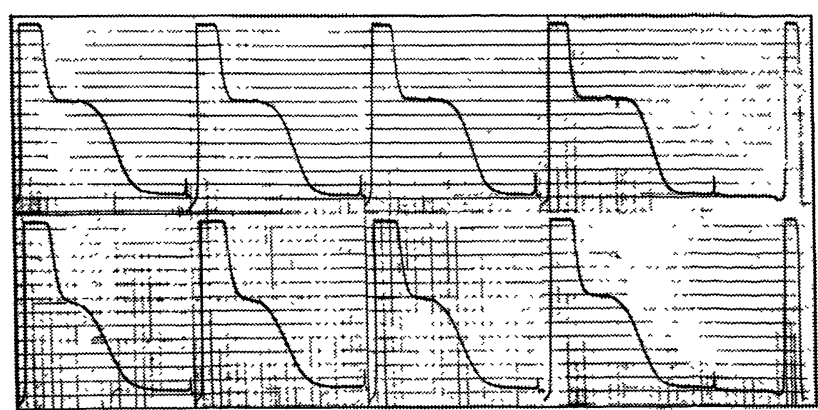

FIG. 9. - Enregistrements pour une détermination de constante de sédimentation.

Substance : Histone F1-CF3.

Vitesse : $52640 \mathrm{t} / \mathrm{mn}$.

Température : $20^{\circ} \mathrm{C}$.

Longueur d'onde : $275 \mathrm{~nm}$.

Les enregistrements successifs sont présentés de droite à gauche et de haut en bas : les clichés 1 et 5 montrent la totalité de l'image de la cellule; les autres clichés sont limités à la partie utile de la cellule comprise entre le ménisque et la ligne de repère extérieur. L'intervalle de temps séparant 2 clichés successifs est de $16 \mathrm{mn}$.

coefficient de diffusion et un enregistrement d'un équilibre de sédimentation.

Les valeurs de $S_{w-20}, D_{w-20}$ et $M_{w}$ ont permis de préciser les caractéristiques morphologiques de cette protéine et d'établir que cette fraction d'histone est monodisperse [6].

3. ETUde D'UNE ENZYME présentant un phénomène réversible d'association en fonction de la concentration.

L'enzyme « Glutamate Déhydrogénase " (E. C. 1.4. 1.3) ou G. D. H. de foie de bœuf a été étudié par équilibre de sédimentation à des concentrations com-

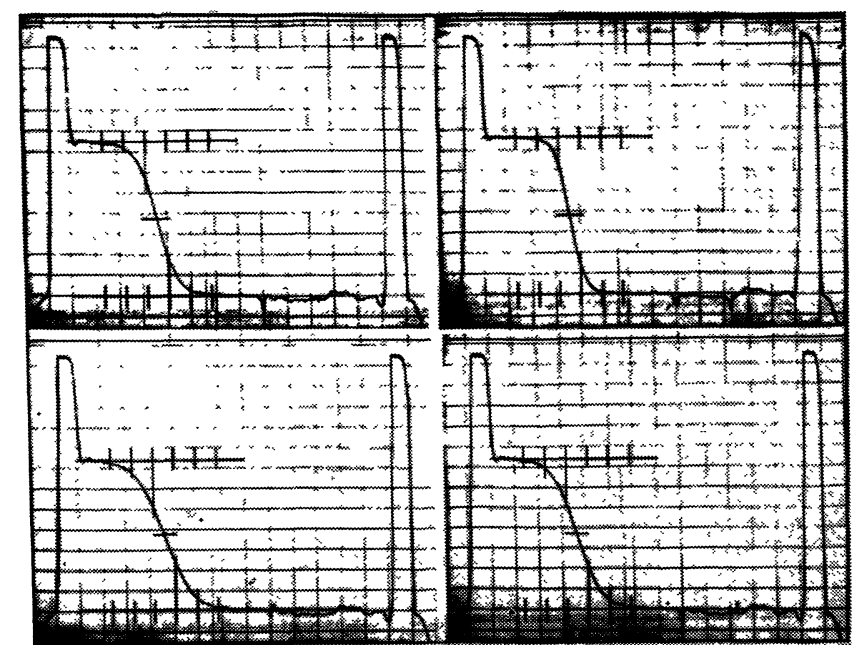

Fig. 10. - Mesure de diffusion brownienne.

Substance : Histone F1-CF3.

Vitesse : $6166 \mathrm{t} / \mathrm{mn}$.

Température : $20^{\circ} \mathrm{C}$.

Longueur d'onde : $275 \mathrm{~nm}$.

Les enregistrements ont été faits respectivement à 77,109 , 141 et $173 \mathrm{mn}$ (de droite à gauche et de haut en bas). La durée d'exploration de l'image est de $15 \mathrm{~s}$.

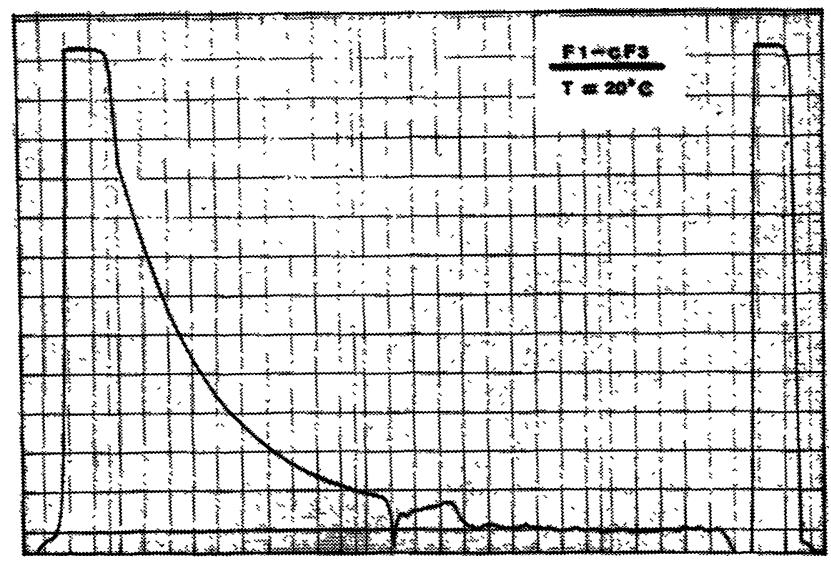

FIg. 11. - Enregistrement d'un équilibre de sédimentation. Substance : Histone F1-CF3.

Vitesse : $19160 \mathrm{t} / \mathrm{mn}$.

Température : $20^{\circ} \mathrm{C}$.

Longueur d'onde : $275 \mathrm{~nm}$.

Densité optique initiale $(275 \mathrm{~nm}-1,2 \mathrm{~cm}): 0,420$.

La durée de balayage de l'image est de $30 \mathrm{~s}$.

prises entre $0,010 \times 10^{-3} \mathrm{~g} / \mathrm{ml}$ et $2,0 \times 10^{-3} \mathrm{~g} / \mathrm{ml}$. Ce domaine de concentration a été couvert par les mesures de densité optique à 295,280 et $230 \mathrm{~nm}$ en cellule double secteur de 12 et $30 \mathrm{~mm}$ d'épaisseur. La valeur de la masse moléculaire moyenne en poids $M_{w}$ de l'oligomètre actif a pu être déterminée à concentration nulle : la valeur obtenue $M_{w}=3,12 \times 10^{5} \pm 0,1 \times 10^{5}$ est en parfait accord avec la valeur donnée par diffusion de la lumière [7].

Les différentes valeurs moyennes de la masse moléculaire ont été calculées suivant Roark et Yphantis [8].

Dans un domaine de concentrations suffisamment 
faibles pour pouvoir négliger la contribution du second coefficient du viriel, le calcul de la variation de $M_{w}$ en fonction de la concentration, à partir des équilibres de sédimentation, est en très bon accord avec les valeurs observées par diffusion de la lumière et permet de conclure que le processus d'association réversible de la G. D. H. est parfaitement décrit par un modèle d'association linéaire avec une seule constante d'association.

Le calcul de $M_{n}$ et de $M_{z}$ confirme ce résultat bien que pour les valeurs de $M_{\mathrm{z}}$ l'accord soit moins bon, quoiqu'encore satisfaisant : il apparaît nettement que la mesure des densités optiques n'est pas encore suffisamment précise [9].

4. ETude des INTERACTIONS ADN-Cytochrome C. Ce système a été choisi pour développer et mettre au point la technique d'étude des interactions ADNprotéines et plus spécialement ADN-histones : le cytochrome $\mathrm{C}$, en effet, est une protéine basique, bien connue, et qui a l'avantage de posséder un chromophore à $410 \mathrm{~nm}$ dans une région spectrale où l'ADN n'absorbe pas.

L'ADN utilisé était segmenté par irradiation aux ultrasons et présentait les caractéristiques suivantes :

$$
\begin{aligned}
S_{w-20} & =7,8 \\
M / L & =210 \text { dalton } \AA^{-1} \\
\text { Hyperchromicité } & =42 \% .
\end{aligned}
$$

L'étude de ce système n'a pu être faite par équilibre de sédimentation et nous avons été contraints d'effectuer l'étude stœchiométrique par vitesse de sédimentation: le calcul des constantes de sédimentation moyennes de l'ADN, $S_{p}$, et du cytochrome C, $S_{A}$, a été effectué par la méthode du transport et les résultats ont été interprétés suivant Steinberg et Schachman [10].

Cette technique s'est avérée très délicate à mettre en œuvre par suite de la précision élevée requise dans la mesure des densités optiques et ceci bien que nous soyons dans des conditions favorables du fait que la bande d'absorption du cytochrome C est située largement en dehors de celle de l'ADN.

Enfin le temps de dépouillement manuel des enregistrements est excessivement long par rapport à la durée réelle des expériences: une sédimentation, réalisée avec le circuit multiplexeur (3 rapports différents $\mathrm{ADN} /$ Cytochrome C) et enregistrée à 260 et $410 \mathrm{~nm}$, nécessite, à raison d'un strict minimum de 30 points par courbe, le relevé et la perforation sur cartes de 3600 valeurs de densité optique. Une étude stœchiométrique complète requiert évidemment un grand nombre de rapports ADN/Cytochrome C différents. Nous avons dû nous limiter à 6 valeurs différentes de ce rapport.

Si ce travail n'a pas permis de déterminer avec précision les quantités de cytochrome C lié et libre à partir des valeurs $\overline{S_{p}}$ et $\bar{S}_{A}$, il a fourni cependant d'intéressants résultats concernant la formation du complexe, la stœchiométrie et l'initiation de la fixation du cytochrome $\mathrm{C}$ à partir des extrémités du polymère [11].

Conclusion. - Le spectrophotomètre double faisceau à balayage d'image, construit au C. R. M., s'est montré d'un emploi sûr, reproductible et précis.

L'expérience acquise durant deux années d'utilisation quotidienne permet d'établir un bilan d'exploitation favorable, mais nous devons émettre quelques remarques :

- la précision des valeurs de densité optique est liée avant tout à la linéarité et à la stabilité du transducteur logarithmique : le transducteur utilisé est nettement supérieur au transducteur à diodes, mais c'est un transducteur analogique dont la précision sera toujours limitée ;

- le recyclage synchrone des voltmètres de crête s'est révélé indispensable pour un tracé précis des courbes de distribution et permet des enregistrements rapides sans perte ni distortion de l'information.

Le système, tel qu'il a été conçu par $\mathrm{H}$. Schachman et tel que nous l'avons réalisé nous-mêmes, est un spectrophotomètre double faisceau : cependant il ne faut pas oublier que la fente d'exploration placée devant le photomultiplicateur a une largeur faible et que cette largeur, rapportée au niveau de la cellule de mesure, devient effectivement très petite $(30 \mu$ dans notre cas) : de microscopiques inhomogénéités optiques sur les fenêtres en quartz au niveau de chacun des secteurs de la cellule qui, dans le cas du faisceau large d'un spectrophotomètre classique (8 à $10 \mathrm{~mm}$ sur 3 à $4 \mathrm{~mm}$ ), seraient sans influence, prennent ici une certaine importance en perturbant différemment les impulsions correspondant aux deux secteurs; ceci conduit à des accidents, minimes, dans les courbes enregistrées mais qui affectent la précision des mesures, surtout dans le cas des équilibres de sédimentation. La correction d'erreur de ce type ne peut se faire valablement que par un traitement sur ordinateur.

Aussi dans le but d'accroître les possibilités d'un tel système, avons-nous entrepris la construction d'un nouveau type de spectrophotomètre à sortie digitale qui permettra une acquisition automatique des valeurs des densités optiques et autorisera un traitement complet des données de l'ultracentrifugation, technique très puissante pour l'étude et la compréhension des interactions entre biopolymères.

Remerciements. - Nous exprimons toute notre gratitude à $\mathrm{M}$. le Professeur Sadron pour l'intérêt qu'il a manifesté lors de l'étude de ce projet et pour la confiance et l'aide précieuse qu'il nous a accordées lors de sa réalisation.

La partie mécanique de ce spectrophotomètre est l'œuvre de M. Lentz, mécanicien.

Nous devons remercier $M$. Lœb qui a collaboré momentanément à l'élaboration du projet.

Cette réalisation a bénéficié d'une aide de la D. G. R. S. T. (convention 63-FR-246). 


\section{Bibliographie}

[1] Hanlon (S.), Lamers (K.), Lauterbach (G.), JohnSON (R.), Schachman (H. K.), Arch. Biochem. Biophys., 1963, 99, 157.

[2] Lamers (K.), Putney (F.), Steinberg (I. Z.), SchachMAN (H. K.), Arch. Biochem. Biophys., 1963, 99, 379.

[3] Gianelli (G.), Stanchi (L.), Nuclear Inst. and Methods, 1960, 8, 79.

[4] Vautrin (J. P.), Thèse $3^{\text {e }}$ Cycle ( Applications des amplificateurs logarithmiques à la discrimination de particules chargées, détectées par semi-conducteurs ), 1966, Strasbourg.

[5] M. I. T. Radiation Laboratory Series. Volume 19
(( Waveforms )) Chapitre 14, page 508. Mc GrawHill Book Cy N. Y., 1949.

[6] Champagne (M.), Pouyet (J.), Ouellet (L.), Garel (A.), Bull. Soc. Chimie Biol., 1970, 52, 377.

[7] Eisenberg (H.), Tomkins (G. M.), J. Mol. Biol., 1968, 31, 37.

[8] Roark (D. E.), Yphantis (D. A.), Ann. N. Y. Acad. Sci., 1969, 164, 245.

[9] Reisler (E.), Pouyet (J.), Eisenberg (H.), Biochemistry, 1970, 9, 3095.

[10] Steinberg (I. Z.), Schachman (H. K.), Biochemistry, 1966, 5, 3728.

[11] Cremel (G.), Thèse $3^{\mathrm{e}}$ cycle (" Etude d'un complexe ADN-Cytochrome C ). Strasbourg, 1969. 